\title{
Patterning counts: Individual differences in children's calculation are uniquely predicted by sequence patterning
}

\author{
Kelsey J. MacKay, Bert De Smedt* \\ Faculty of Psychology and Educational Sciences, KU Leuven-University of Leuven, B-3000 Leuven, Belgium
}

\section{A R T I C L E I N F O}

\section{Article history:}

Received 26 October 2017

Revised 4 April 2018

Available online 8 September 2018

\section{Keywords:}

Patterning

Arithmetic

Calculation

Symbolic magnitude processing

Children

Sequence patterns

\begin{abstract}
A B S T R A C T
Many studies have examined the cognitive determinants of children's calculation, yet the specific contribution of children's patterning abilities to calculation remains relatively unexplored. This study investigated whether children's ability to complete sequence patterns (i.e., add the missing element into 2-4-?-8) uniquely predicted individual differences in calculation and whether these associations differed depending on the type of stimuli in these sequence patterns (i.e., number, letter, time, or rotation). Participants were 65 children in first and second grade $\left(M_{\mathrm{age}}=7.40\right.$ years, $\left.S D=0.44\right)$. All children completed four tasks of sequence patterning: number, letter, time, and rotation. Calculation was measured via addition and subtraction tasks. We also measured cognitive determinants of individual differences in calculation-namely symbolic number comparison, motor processing speed, visuospatial working memory, and nonverbal IQ-to verify whether patterning predicted calculation when controlling for these additional measures. We observed significant relationships between the patterning dimensions and calculation, except for the rotation dimension. Follow-up regressions, controlling for the aforementioned cognitive determinants of calculation, revealed that the number and time dimensions were strong predictors of calculation, whereas the evidence for the letter dimension was only anecdotal and the evidence for the rotation dimension was nonexistent, suggesting some degree of specificity of different types of sequence patterning in predicting calculation. Symbolic magnitude processing remained a powerful unique correlate of calculation performance. These findings add to our understanding of
\end{abstract}

* Corresponding author.

E-mail address: bert.desmedt@kuleuven.be (B. De Smedt). 
individual differences in calculation ability, such that sequence patterning could begin to be considered as one of the cognitive skills underlying calculation ability in young children.

(c) 2018 Elsevier Inc. All rights reserved.

\section{Introduction}

Many studies have examined the cognitive determinants of children's calculation, yet the specific role of children's (sequence) patterning remains relatively unexplored. Even though there have been suggestions of the links between patterns and mathematics (Papic, Mulligan, \& Mitchelmore, 2011; Starkey, Klein, \& Wakeley, 2004; Warren, Cooper, \& Lamb, 2006), and patterning remains a component of children's education (Clements, Sarama, \& Liu, 2008), research on patterning as one of the potential components of individual differences in mathematics development is lacking, with the existing literature on the origins of individual differences in mathematics being mainly focused on working memory or numerical processing (Peng, Namkung, Barnes, \& Sun, 2016; Schneider et al., 2017; Vanbinst \& De Smedt, 2016). While the existing body of patterning studies have almost exclusively examined the association between repeating patterns and calculation (Miller, Rittle-Johnson, Loehr, \& Fyfe, 2016; Papic et al., 2011), sequence patterning is a relatively understudied type of patterning in comparison with repeating patterns. Therefore, the goal of the current study was to address this gap in the literature and examine the specific role of sequence patterning in elementary school children's calculation. In the remainder of the Introduction, we summarize the existing literature on relational thinking and patterning and present the aims of the current study.

Sequence patterns, compared with other types of patterning (e.g., repeating patterns), might be more critical to children's mathematical development when referring to counting sequences and number representation (e.g., counting; Thomas, Mulligan, \& Goldin, 2002). The reason for this could be due to, for example, sequence patterns being related to relational thinking. Relational thinking, in the context of mathematical cognition, refers to the idea of inspecting the connections between two or more objects to solve a problem (Molina, Castro, \& Ambrose, 2005). Although repeating patterns would also activate this type of thinking, sequence patterns are a more complex method by which to examine children's relational thinking. Taking relational thinking into account, as well as the complexity of sequence patterns compared with repeating patterns, sequence patterns are a potentially more beneficial type of patterning to examine compared with repeating patterning. Moreover, the idea of relational thinking has been applied in the domain of algebra (Carpenter, Levi, Franke, \& Zeringue, 2005) and fraction learning (Empson, Levi, \& Carpenter, 2011) and has been shown to be an important step in the development of arithmetic to algebraic learning (Napaphun, 2012). Sequence patterning, therefore, can be investigated with regard to its importance in mathematics competency, potentially through the mechanism of relational thinking.

Although relational thinking can be conceived as a potential general mechanism in sequence patterning, it is also possible that relational thinking is only involved in more specific associations with sequence patterning. For instance, a sequence pattern can be in numbers (i.e., 2-4-6-8) but can also be in letters (i.e., a-c-e-g). The question regarding relational thinking in sequence patterns, therefore, can also include the distinction between domain-specific sequence patterns (i.e., sequence patterns are related to mathematics only within some of these dimensions) and domain-general sequence patterns (i.e., every dimension of sequence patterning is related to mathematics). In examining distinct dimensions of sequence patterning and their relations to mathematics, the potential mechanism of relational thinking in the association between sequence patterning and mathematics can be examined as being either domain specific or domain general.

A pattern is any replicable regularity in which the items have relations to one another and are predictable (Economopoulos, 1998; Fyfe, McNeil, \& Rittle-Johnson, 2015; Hendricks, Trueblood, \& Pasnak, 2006; Papic et al., 2011). Patterns can be found in a variety of domains, including (but not limited to) 
poetry, music, and mathematics (Björklund \& Pramling, 2014), showing that it is important to understand the placement and use of patterning skills.

The type of patterning that is usually the focus of the classroom, as well as the literature on children's mathematical development, is that of repeating patterns (e.g., a-b-a-b-a-b; Clements et al., 2008; Rittle-Johnson, Fyfe, McLean \& McEldoon, 2013), and these repeating patterns are deemed important for later mathematical development (Economopoulos, 1998; Ford \& Crew, 1991; Rittle-Johnson, Fyfe, Loehr, \& Miller, 2015). However, much less focus is given to sequence patterning. Piaget (1952) theorized that a milestone in children's cognitive development lies in seriation and transitivity-in other words, sequences and patterns-showing that it is a relevant area for research on children's mathematical development. Sequence patterns can also be linked to an understanding of ordinality, such that it involves participants providing yes/no answers regarding a set of numbers appearing in a row. Importantly, ordinality has been found to be linked to various aspects of mathematical ability, specifically the processing of symbolic numbers (Goffin \& Ansari, 2016; Lyons, Vogel, \& Ansari, 2016). Furthermore, both repeating and sequence patterns offer a method by which children can exercise their relational thinking, as mentioned above, to understand how items in a pattern relate to one another (Blanton \& Kaput, 2005; Pasnak et al., 2015). Taken together, these arguments motivated the use of sequence patterning in the current study.

A sequence pattern is defined as a type of pattern in which the items follow a certain sequence that is not repeating but rather is increasing or decreasing at a constant rate and is sometimes referred to as a growing pattern (Economopoulos, 1998; Papic et al., 2011). As an example, a sequence pattern involving numbers would look like this: $2-4-6-8-10$. In some tasks using sequence patterning, a sequence is presented with one of the numbers missing (i.e., 2-4-?-8-10) and children are then presented with four options from which to choose, one of which is the correct answer (Gadzichowski, 2012).

Previous intervention studies (Hendricks et al., 2006; Kidd et al., 2013, 2014) have shown that patterning instruction involving a variety of patterns (e.g., repeating, symmetrical increasing sequence patterns) in many dimensions (e.g., numbers, letters, shapes, symbols, objects) improves academic achievement in mathematics as compared with control groups receiving other types of instruction (e.g., math, reading, social studies) or business-as-usual instruction. These studies provide evidence that relationships between patterning and mathematical ability exist. However, these findings were based on the combination of all types of patterns, including sequence patterns, in all dimensions, leaving the disentanglement of which type(s) of patterning and in which dimensions contribute to these findings.

Lee, Ng, Bull, Pe, and Ho (2011), examined the role of sequence patterning in 10-year-old children's mathematical abilities via a number series task (sequences of whole or rational numbers following a rule) and a function machine task (pairs of numbers with a specific relationship). They observed unique associations between the patterning tasks and different measures of mathematical ability (i.e., computational fluency and algebraic proficiency). However, their patterning tasks included only numerical stimuli, leaving it unresolved as to whether only numerical patterning abilities or more general patterning abilities are predictive of mathematical performance.

In a follow-up study, Lee et al. (2012) investigated the same relationships but in younger children (6-year-olds). They administered executive functioning tasks, numerical and arithmetic proficiency measures, and three types of patterning tasks. They administered a similar function machine task, as described above, with both geometric shapes and numbers. They also included a shapes-withinshapes patterning measure, which involved manipulating the size, shape, and position of the shapes

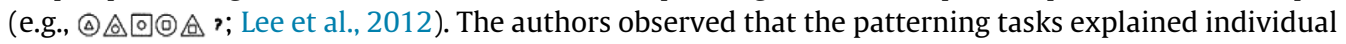
differences in numerical and arithmetical proficiency and that the numerical patterning task explained higher variance compared with the geometrical task and shapes-within-shapes task. Although Lee et al. (2012) made an important step in understanding the role of sequence patterns, they did not consider other specific determinants of individual differences in mathematics, such as number processing, as we do in the current study.

More recently, Pasnak et al. (2016) examined the specific relationship between sequence patterning specifically and mathematical abilities in first graders. The results showed that children's fall scores on number sequences were significantly correlated with their spring scores on the standardized math 
assessment, whereas the fall scores on the standardized math assessment were not significantly correlated with spring scores on number sequences. However, one limitation of this study, much like many other patterning studies (e.g., Hendricks et al., 2006; Kidd et al., 2013, 2014), is the limited specificity regarding mathematical abilities. In other words, these studies used general, standardized mathematical measures that examined large components of mathematical ability (e.g., quantitative concepts, mathematical concepts), leaving it unresolved as to which type of mathematical ability sequence patterning is specifically related. Because predictors of individual differences are dependent on the math ability under study (De Smedt, 2016; Peng et al., 2016; Schneider et al., 2017), the current study restricted the focus to calculation (i.e., addition and subtraction) because it plays a quintessential role in mathematics and given that the existing body of literature has largely focused on this part of mathematics.

\section{The current study}

As hinted briefly in the literature summary of patterning studies, there are two major shortcomings in previous patterning research that the current study aimed to resolve. First, previous patterning research has not investigated different dimensions of sequence patterning, leaving it unresolved whether sequence patterning generally relates to mathematics or whether this association is domain specific (i.e., number patterns relate to mathematics, whereas letter patterns do not). Second, previous research examining the major determinants of individual differences in calculation have not considered sequence patterning as a potential major determinant; therefore, it is unknown whether sequence patterning uniquely contributes to individual differences in calculation beyond other known cognitive determinants of calculation ability such as numerical magnitude processing and visuospatial working memory (De Smedt et al., 2009; Peng et al., 2016; Schneider et al., 2017; Vanbinst \& De Smedt, 2016).

Based on these two shortcomings in patterning research, two research questions were derived. The first was to determine the associations between dimensions of the sequence patterning task and calculation. If the association is domain specific, we expected to see correlations between the number and time dimensions (given their proximity to number) and calculation, whereas if the association is general, we expected to see correlations between each of the sequence patterning dimensions and calculation. The second question was to determine whether sequence patterning plays a unique role in explaining the individual differences in children's calculation when symbolic number comparison, motor processing speed, visuospatial working memory, and nonverbal IQ were taken into account. To follow up previous patterning literature (Hendricks et al., 2006; Kidd et al., 2013, 2014; Pasnak et al., 2016), we focused on children in early elementary school.

To answer these two questions, we administered a sequence patterning task in four dimensions (number, letter, time, and rotation) and a calculation task assessing both addition and subtraction. In addition, to assess how patterning uniquely contributes to calculation skills, various cognitive measures were added because the literature clearly shows that individual differences in arithmetic can be explained by a variety of cognitive skills (Chu, vanMarle, \& Geary, 2015; Desoete, 2015; Xenidou-Dervou, De Smedt, van der Schoot, \& van Lieshout, 2013). In terms of cognitive measures, we included numerical magnitude processing because it is known to be crucial in explaining the individual differences found in children's calculation performance (Vanbinst \& De Smedt, 2016), but we restricted our focus to symbolic measures in view of the meta-analysis by Schneider et al. (2017) showing that symbolic measures are significantly more correlated to individual differences in mathematics than nonsymbolic ones. We also focused on visual measures for working memory and IQ given that the patterning task was also visual in nature. Visuospatial working memory has also been found to be related to calculation (e.g., De Smedt et al., 2009; Schmerold et al., 2017), further motivating its use in the current study. Against this background, we assessed children's symbolic number comparison, motor processing speed, visuospatial working memory, and nonverbal IQ in addition to sequence patterning as potential variables that could explain variance in children's calculation ability. 


\section{Method}

\section{Participants}

Participants were 65 typically developing children, ranging in age from 6 to 9 years (28 girls and 37 boys; $M_{\mathrm{age}}=7.40$ years, $S D=0.44$ ), from three different international schools in Belgium. Of this sample, 25 children were recruited from two of the schools during the spring and 40 children were recruited from the third school during the following fall. Grouping all the children together, 15 were in Grade 1 (23\%) and 50 were in Grade 2 (77\%). All children were taught in English and followed similar curricula. All students were able to communicate well in English, follow the task instructions, and ask questions clearly if help was needed.

\section{Materials}

\section{Patterning}

This task was the same as in Kidd et al. (2014) and consisted of 12 problems in each of the four dimensions of patterns (i.e., number, letter, time, and rotation) for a total of 48 items. The items were all combined, regardless of dimension, as decided by a Latin square table. There were four practice items (one for each dimension). The patterns consisted of five stimuli with one item missing, as shown by a question mark. The items varied such that the first, third, or fifth item of the pattern could be missing. Depending on whether the item was vertically or horizontally presented, four possible answers were presented beside or below the problem, respectively. Children received a maximum score of 12 for each of the four dimensions of patterns. For an example of each of the dimensions of patterns, see Fig. 1.

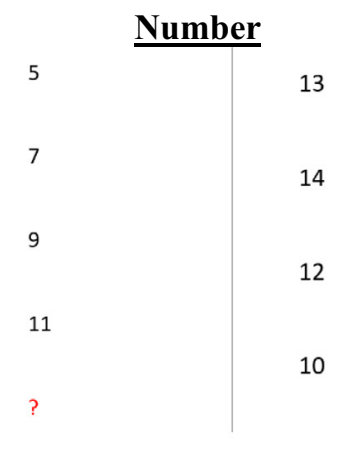

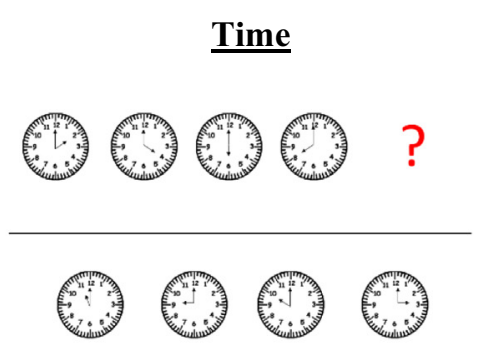

$\underline{\text { Rotation }}$

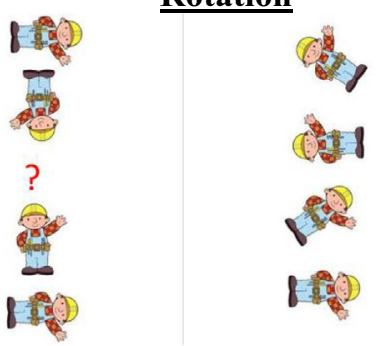

Fig. 1. Examples of each of the dimensions of patterns used in the patterning task, based on Gadzichowski (2012). From left to right and from top to bottom, an example of number patterns, letter patterns, time patterns, and rotation patterns can be seen. 


\section{Calculation}

This task comprised six sections, each increasing in difficulty. There were two versions, one for addition and one for subtraction, for a total of 12 subsections. The sections were lettered and were categorized based on the sum and minuend of the problems for the addition and subtraction versions, respectively. The sections were made to increase in difficulty with two assumptions: that smaller sums and minuends were easier and that problems where children were not required to carry were easier than those where children were required to carry. The items increased from single to double to triple digits, with each set first presenting no-carry problems followed by carry problems. Each section contained six problems and had approximately an equal number of problems in each decade. Problems that contained a decade, 0 , or 1 in either the operands or solutions were excluded. A list of the specific problems is provided in the Appendix. The children moved through the sections at their own pace and were instructed to continue through as many sections as possible. There was no time limit. The total number of problems answered correctly, per operation, was used as the score.

\section{Symbolic number comparison}

We administered the SYMP Test (Brankaer, Ghesquière, \& De Smedt, 2017) to assess children's symbolic magnitude comparison skills. Children's task was to cross out, as fast and accurately as possible, the largest number when given a pair of numbers. The task existed in two versions, single digit and double digit, each comprising four practice problems followed by 60 problems, of which children completed as many as possible in $45 \mathrm{~s}$. Children received a score for the total number of problems correct for each version of the task. Performance on this group-administered test is known to correlate very highly with classic computerized versions of the comparison task in children of this age (rs > .58; Brankaer et al., 2017).

\section{Motor processing speed}

This measure was taken from the SYMP Test (Brankaer et al., 2017) and was administered as a control task to account for the speed with which children completed the symbolic number comparison tasks. It had exactly the same design as the SYMP Test (four practice items followed by 60 items) except that the stimuli were pairs of shapes that were either solid black or white with a black outline. Children were asked to cross out the black shape. Children received one score for the total number of problems correct within the time limit (20 s).

\section{Visuospatial working memory}

The Corsi Span measure was used to assess visuospatial working memory (De Smedt et al., 2009). The experimenter placed a wooden plate to which nine blocks at random positions were glued. The experimenter indicated to children that she would tap a sequence of blocks and their task was to repeat the tapped sequence in the same order. After two practice problems, the test stimuli began, starting with three sequences of two blocks. The sequence increased with one block after three items of the same list length. The task stopped when children could not complete all three problems of the same length. Children's score was calculated to be the total number of items solved correctly.

\section{Nonverbal IQ}

We used the block design task from the Wechsler Intelligence Scale for Children-third edition (WISC-III; Wechsler, 1991) as a proxy for nonverbal IQ.

\section{General procedure}

The testing order was the same for every child. Children first completed the group-administered tests in the following order: patterning task, calculation task, single-digit number comparison, motor processing speed, and double-digit number comparison. After that, the visuospatial working memory task and nonverbal IQ task were administered individually in a quiet room. 


\section{Results}

\section{Descriptive statistics}

Descriptive statistics for all the tasks and their reliabilities can be found in Table 1 . The addition and subtraction items of the calculation task were highly positively correlated $(r=.64, p<.001)$, as were the single- and double-digit number comparison scores $(r=.70, p<.001)$; therefore, they were combined into a total calculation score and a total number comparison score, respectively, to reduce the number of correlations. In addition, the original patterning task from Kidd et al. (2014) had 48 items, but we discovered an error in one rotation item after testing; therefore, this item was removed from the analysis, resulting in 11 items instead of 12 for this dimension. Results show no ceiling or floor effects, with each task having high reliability with the exception of the rotation dimension, which was found to have a lower reliability.

\section{Correlational analyses}

A summary of the correlations between each of the measures can be found in Table 2. All patterning dimensions except rotation were positively significantly correlated with children's calculation. These relationships can be seen graphically in Fig. 2. The number and time dimensions significantly positively correlated with children's total number comparison score. The letter and time dimensions significantly positively correlated with children's visuospatial working memory. None of the patterning dimensions correlated with motor processing speed. Only the rotation dimension of the patterning test correlated significantly with children's nonverbal IQ. Age correlated significantly positively only with the letter dimension of the patterning task, as well as motor speed and visuospatial working memory, whereas sex significantly correlated only with number comparison. Finally, it should be noted that all the patterning dimensions significantly correlated with each other, with the exception of the correlation between the letter and rotation dimensions.

\section{Linear regressions}

Four linear regressions were executed to examine how each of the dimensions of the patterning tasks uniquely predicted children's calculation scores. Other cognitive determinants of children's calculation were added to determine whether the dimensions contributed additional explained variance in children's calculation, namely symbolic number comparison, motor processing speed, visuospatial working memory, and nonverbal IQ. Age was added in the linear regressions because it was significantly correlated with the letter dimension of the patterning task. Sex was not added as a predictor

Table 1

Means, standard deviations, minimum, maximum, theoretical maximum, and reliability for all tasks completed.

\begin{tabular}{|c|c|c|c|c|c|c|}
\hline & $M$ & $S D$ & Min & Max & Theoretical Max & Reliability \\
\hline \multicolumn{7}{|l|}{ Patterning task } \\
\hline Number & 7.34 & 3.71 & 0 & 12 & 12 & $.88^{\mathrm{a}}$ \\
\hline Letter & 5.95 & 3.31 & 0 & 11 & 12 & $.81^{\mathrm{a}}$ \\
\hline Time & 6.11 & 3.10 & 0 & 12 & 12 & $.77^{\mathrm{a}}$ \\
\hline Rotation & 4.05 & 2.18 & 0 & 9 & 11 & $.54^{\mathrm{a}}$ \\
\hline Calculation task total & 33.06 & 14.44 & 11 & 71 & 72 & $.96^{\mathrm{a}}$ \\
\hline Symbolic number comparison total & 43.35 & 9.84 & 19 & 69 & 120 & $.79^{\mathrm{b}}$ \\
\hline Motor processing speed & 25.98 & 6.41 & 12 & 42 & 60 & $.86^{\mathrm{b}}$ \\
\hline Visuospatial working memory & 8.84 & 1.99 & 5 & 14 & 24 & $.77^{\mathrm{c}}$ \\
\hline Nonverbal IQ & 26.15 & 11.59 & 2 & 51 & 69 & $.87^{\mathrm{d}}$ \\
\hline
\end{tabular}

a Cronbach's alpha calculated from the current study.

b Test-retest reliability from the manual in children of the same age.

c Calculated from the sample in De Smedt et al. (2009) in children of the same age.

d From Sattler (2001). Nonverbal IQ uses raw scores. 
Table 2

Zero-order correlations of all tasks used.

\begin{tabular}{|c|c|c|c|c|c|c|c|c|c|c|c|}
\hline & 1 & 2 & 3 & 4 & 5 & 6 & 7 & 8 & 9 & 10 & 11 \\
\hline 1. Number & - & & & & & & & & & & \\
\hline 2. Letter & $.61^{* *}$ & - & & & & & & & & & \\
\hline 3. Time & $.51^{* *}$ & $.36^{* *}$ & - & & & & & & & & \\
\hline 4. Rotation & $.36^{* *}$ & .14 & $.40^{* *}$ & - & & & & & & & \\
\hline 5. Calculation & $.53^{* *}$ & $.37^{* * *}$ & $.54^{* *}$ & .18 & - & & & & & & \\
\hline 6. Number comparison & $.31^{*}$ & .18 & $.39^{* * *}$ & .07 & $.56^{* *}$ & - & & & & & \\
\hline 7. Motor speed & .15 & -.07 & .22 & .13 & $.36^{* *}$ & $.44^{* *}$ & - & & & & \\
\hline 8. Visuospatial working memory & .20 & $.28^{*}$ & $.29^{*}$ & .19 & $.33^{* *}$ & .15 & .08 & - & & & \\
\hline 9. Nonverbal IQ & .05 & .20 & .23 & $.34^{* *}$ & .11 & .04 & -.03 & .23 & - & & \\
\hline 10. Age & .16 & $.26^{*}$ & .14 & .14 & .24 & .10 & $.28^{*}$ & $.27^{\circ}$ & .17 & - & \\
\hline 11. Sex & .09 & .21 & -.01 & -.00 & -.16 & $-.42^{* *}$ & -.09 & -.12 & -.21 & .22 & - \\
\hline
\end{tabular}

* $p<.05$.

$p<.01$.

A

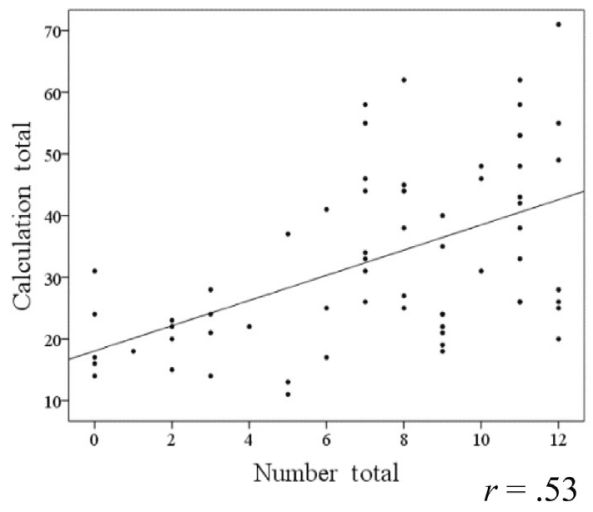

C

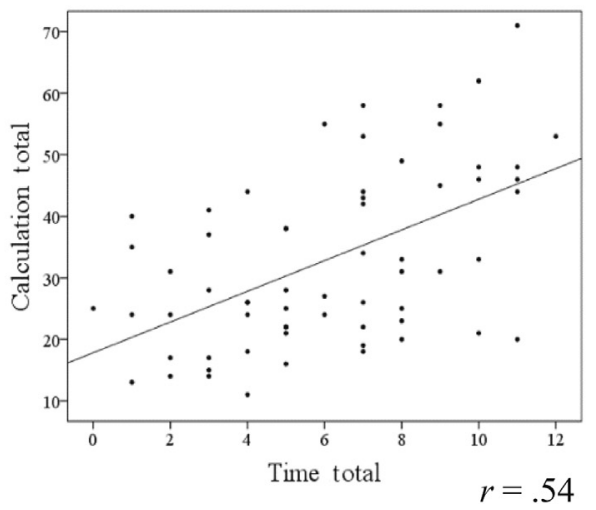

B

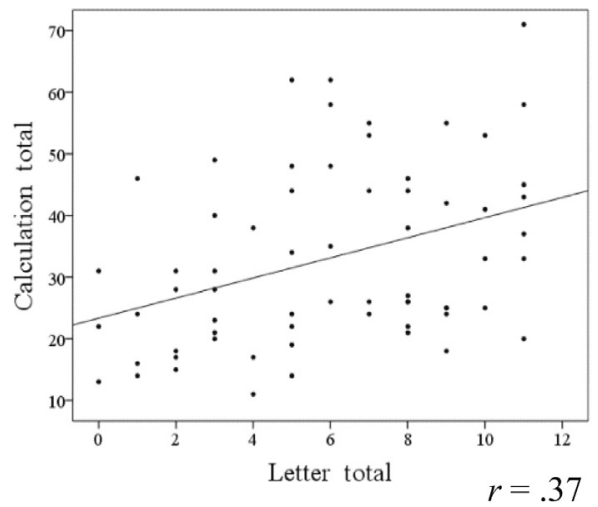

D

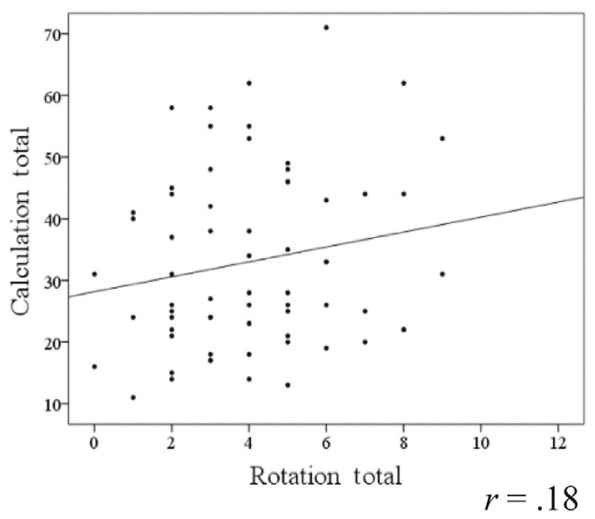

Fig. 2. Linear relationships between each dimension of the patterning task and the total calculation score. 
because it showed no significant correlations with any of the dependent variables (Table 2). The children in this study came from three different schools; therefore, school was first entered as a dummy variable to account for school-to-school differences. The results of the regression analyses were similar whether or not accounting for school; therefore, only the results without the dummy variables are reported.

The outcome of the regression analyses is depicted in Table 3. It is important to note that the regression diagnostics (variance inflation factor) indicated that there was no evidence for multicollinearity of the predictors. The regression analyses indicated that the number, time, and letter dimensions were significant unique predictors of children's calculation when controlling for age, symbolic number comparison, motor processing speed, visuospatial working memory, and nonverbal IQ, whereas the rotation dimension was not. Symbolic number comparison emerged as a powerful unique predictor even when controlling for patterning abilities (in the different dimensions).

We also ran Bayesian analyses that allowed us to test the degree of support for a given predictor. We calculated the Bayes factor, which is the ratio of the evidence for the alternative hypothesis compared with the null (see Andraszewicz et al., 2015, for an elaboration). Adding the Bayes factors to our analyses (Table 3) allowed us to infer which hypothesis for a given predictor is the most plausible (i.e., alternative vs. null) and to identify the predictors for which the evidence in the data is the strongest. The Bayesian analyses were generally in line with the frequentist data, supporting the observation that the number and time dimensions were strong predictors of the variability in children's calculation. The evidence for the contribution of the letter dimension was only anecdotal. For the rotation dimension, there was more evidence in the data for the null hypothesis of no association, although this evidence was also only anecdotal. Symbolic number comparison was an even stronger predictor (as

Table 3

Linear regression models predicting calculation, estimated for the different dimensions of the patterning task.

\begin{tabular}{|c|c|c|c|c|c|c|}
\hline Predictor & Adjusted $R^{2}$ & $\beta$ & $p$ & $\begin{array}{l}\text { Variance inflation } \\
\text { factor }\end{array}$ & Bayes factor & Unique $R^{2}$ \\
\hline Number $[F(6,54)=9.616, p<.001]$ & .463 & & & & & \\
\hline Number patterning & & .316 & $.003^{* *}$ & 1.138 & 24.001 & .088 \\
\hline Age in months & & .027 & .793 & 1.177 & 0.135 & .001 \\
\hline Symbolic number comparison & & .439 & $.000^{* *}$ & 1.366 & 572.690 & .135 \\
\hline Motor processing speed & & .086 & .435 & 1.323 & 0.340 & .021 \\
\hline Visuospatial working memory & & .116 & .263 & 1.176 & 0.620 & .012 \\
\hline Nonverbal IQ & & .124 & .221 & 1.125 & 0.716 & .014 \\
\hline Letter $[F(6,54)=8.086, p<.001]$ & .415 & & & & & \\
\hline Letter patterning & & .236 & $.037^{*}$ & 1.244 & 2.708 & .044 \\
\hline Age in months & & -.001 & .990 & 1.232 & 0.314 & .000 \\
\hline Symbolic number comparison & & .470 & $.000^{* *}$ & 1.355 & 857.067 & .091 \\
\hline Motor processing Speed & & .147 & .217 & 1.414 & 0.511 & .043 \\
\hline Visuospatial working memory & & .115 & .290 & 1.188 & 0.623 & .011 \\
\hline Nonverbal IQ & & .101 & .344 & 1.150 & 0.564 & .009 \\
\hline Time $[F(6,54)=8.842, p<.001]$ & .440 & & & & & \\
\hline Time patterning & & .301 & $.010^{* *}$ & 1.350 & 13.366 & .067 \\
\hline Age in months & & .055 & .599 & 1.171 & 0.364 & .003 \\
\hline Symbolic number comparison & & .421 & $.001^{* *}$ & 1.451 & 191.537 & .118 \\
\hline Motor processing speed & & .082 & .462 & 1.323 & 0.343 & .018 \\
\hline Visuospatial working memory & & .106 & .318 & 1.190 & 0.501 & .010 \\
\hline Nonverbal IQ & & .058 & .590 & 1.213 & 0.352 & .003 \\
\hline Rotation $[F(6,54)=7.024, p<.001]$ & .376 & & & & & \\
\hline Rotation patterning & & .107 & .337 & 1.164 & 0.633 & .009 \\
\hline Age in months & & .047 & .670 & 1.172 & 0.368 & .002 \\
\hline Symbolic number comparison & & .535 & $.000^{* *}$ & 1.271 & 4299.127 & .217 \\
\hline Motor processing speed & & .067 & .574 & 1.350 & 0.329 & .041 \\
\hline Visuospatial working memory & & .135 & .229 & 1.182 & 0.800 & .015 \\
\hline Nonverbal IQ & & .106 & .348 & 1.209 & 0.657 & .009 \\
\hline
\end{tabular}

${ }^{*} p<.05$.

** $p<.01$. 
indicated by the Bayes factors) than any of the patterning measures. Interestingly, the Bayes factors for age, motor processing speed, visuospatial working memory, and nonverbal IQ all were below 1, indicating that there is more evidence in the data for the null hypothesis (i.e., that this variable is not correlating with calculation) than for the alternative hypothesis.

\section{Discussion}

The goal of the current research was to determine the specific role of sequence patterning in elementary school children's calculation. Two research questions were posed for the current study. First, are associations between dimensions of the sequence patterning task and calculation ability domain specific (i.e., only some dimensions of sequence patterning are associated with calculation) or domain general (i.e., every dimension of sequence patterning is associated with calculation)?. Second, does sequence patterning play a unique role in explaining the individual differences in children's calculation when symbolic number comparison, motor processing speed, visuospatial working memory, and nonverbal IQ are added? The current data revealed that number, letter, and time patterning, but not rotation patterning, correlated with calculation ability. These associations remained when controlling for symbolic number comparison, motor processing speed, visuospatial working memory, and nonverbal IQ. This suggests an important and unique role for sequence patterning in predicting individual differences in calculation, as we elaborate in more detail below.

With regard to the first research question, correlations determined that sequence patterning is significantly positively related to addition and subtraction skills, such that only number, letter, and time patterns were associated with calculation. First, our results extend the literature that has studied the association between repeating patterning and mathematics (Economopoulos, 1998; Ford \& Crew, 1991; Papic et al., 2011; Rittle-Johnson et al., 2015; Starkey et al., 2004) and show that sequence patterning, in certain dimensions (i.e., number, letter, and time), is another type of patterning related to calculation abilities. Therefore, our study also replicated and extended Lee et al. (2012), who found that number patterns were associated with arithmetic proficiency. Furthermore, the results provide evidence that sequence patterning is related to a specific type of mathematical ability, calculation, which extends the findings from previous sequence patterning literature examining broad mathematical measures (Hendricks et al., 2006; Kidd et al., 2013, 2014; Lee et al., 2011, 2012; Pasnak et al., 2016). We focused on calculation as the dependent variable because it plays a quintessential role in mathematics and given that prior research has largely focused on this part of mathematics. With regard to the strength of the correlations, number and time were found to be more strongly related to calculation in comparison with the letter and rotation dimensions, thereby confirming some degree of specificity within sequence patterning.

As hinted above, the rotation dimension of the patterning task did not correlate with individual differences in calculation. Lee et al. (2012) found that numerical patterning explained more variance in numerical proficiency compared with the geometrical task, showing that perhaps geometrical tasks, such as the rotation sequence patterns used in the current study, explain less variance with regard to mathematical abilities. On the other hand, the rotation task was found to have low reliability in the current study, showing that it is possible that the task was too difficult for this age group, thereby resulting in a nonsignificant association between the rotation sequence patterns and calculation. Taking this low reliability of the rotation dimension into account, we should be cautious in drawing strong conclusions about domain specificity. Further investigation is required regarding rotation sequence patterns and their association with calculation in this age group and older.

The similarity in the associations of both number and time with calculation might suggest that perhaps the cognitive representations of sequence patterns in the dimensions of number and time might not be so distant from one another and could even reflect basic magnitudes in different dimensions (e.g., Bueti \& Walsh, 2009; Lourenco \& Bonny, 2017; Park \& Cho, 2017). However, the rotation dimension did not correlate with calculation, suggesting that these representations of space, time, and number might not be so well connected, at least not in children. Future studies are needed to further investigate the interrelations among measures of space, time, and number.

To further examine the possibility of domain specificity within sequence patterning as part of our first aim, the Bayesian regression analyses demonstrated that the association between number and 
calculation was the strongest of all the dimensions on the patterning task. The association between the time dimension and calculation was also found to be quite strong. The evidence for the importance of letter patterns, however, was much less strong, even at the anecdotal level, and there was no evidence for an association between the rotation measure and calculation. In other words, if there were a domain-general association between patterning and calculation, one should observe similar associations for all patterning dimensions under study, and this was not the case. Against this background, the current data suggest that the association between patterning and calculation is more domain specific.

Although our findings point to domain-specific relations between sequence patterning and children's calculation, the underlying mechanisms of this relationship are still unknown. Our results particularly emphasize the role of the number and time dimensions of sequence patterns, rather than the letter and rotation dimensions, in explaining children's calculation, although the Bayesian analyses point to number as being stronger than time. The understanding of the number and time dimensions of sequence patterns could be related to children's understanding of ordinality (i.e., the ability to order numbers correctly). Recently, there has been an increased focus on ordinality and individual differences in mathematical achievement (Goffin \& Ansari, 2016; Lyons \& Beilock, 2011, 2013). However, it should be noted that while sequence patterns and ordinality are similar, they might not tap into the same cognitive abilities. Rather, because sequence patterning requires complex relational reasoning, whereas ordinality tasks do not, the underlying mechanism could perhaps be this relational reasoning with numbers (Carpenter et al., 2005; Empson et al., 2011; Napaphun, 2012) that is used in the sequence patterns, as opposed to the letter and rotation sequence patterns, for which reason number and time are strong predictors of calculation performance.

For our second aim, to understand the unique role of sequence patterning in children's calculation abilities, linear regressions and Bayesian analyses examined the role of each of the dimensions alongside symbolic number comparison, motor processing speed, visuospatial working memory, and nonverbal IQ in explaining variance in children's calculation abilities. These analyses further revealed that the number and time dimensions were unique and stronger predictors of children's calculation ability compared with the letter dimension (anecdotal evidence) and rotation dimension (no evidence). These results are consistent with those of Pasnak et al. (2016) that number sequences, and not letter sequences, are unique predictors of children's calculation abilities, although Pasnak et al. (2016) did not investigate dimensions of time and rotation. Importantly, our findings go beyond Pasnak et al. (2016) by showing that the association with number sequence patterns remains when controlling for other well-known cognitive determinants of calculation.

The current findings also replicate the observation that symbolic number processing is a major underlying ability that predicts children's arithmetic (Schneider et al., 2017; Vanbinst \& De Smedt, 2016). The current data extend this body of evidence by showing that this association also remains when also controlling for sequence patterning abilities, including number patterning. Our data also indicate that numerical magnitude processing and number patterning are related, yet each of them provides a unique contribution in explaining individual differences in calculation. Both symbolic number comparison and sequence patterning tasks require children to use relational reasoning, thereby supporting the idea that relational reasoning could be the underlying mechanism driving both of these competencies. On the other hand, it needs to be emphasized, as is evidenced by the differences in Bayes factors, that the evidential strength for symbolic number comparison as a predictor of calculation was substantially higher than the evidential strength for number or time patterns.

Future research should also examine the relationships between each of the dimensions of sequence patterning and calculation (or other specific mathematical abilities) in a longitudinal study to verify the direction of the association and especially to ensure that our findings generalize beyond our sample of students in international schools. Moreover, the addition of other known cognitive variables, such as approximation skills, could be relevant (Xenidou-Dervou et al., 2013). Furthermore, the current study used increasing sequence patterns, but decreasing sequence patterns have also been used in some patterning research (Hendricks et al., 2006) and would be an interesting next step regarding sequence patterns to determine whether similar associations exist for decreasing sequence patterns. Finally, a future study could examine the same sequence patterning task in older children to get a better sense of the relationships with the rotation dimension given that it could have been too difficult for this age group. 
As for implications of our research, Sarama and Clements (2009) discussed the importance of including (repeating) patterns in daily environments of children. Building on this idea, sequence patterns could also be integrated into the classroom as part of a lesson plan in mathematics. Seeing as our research found that particularly the number and time dimensions of sequence patterns are related to calculation, this activity in math classrooms would be an innovative and potentially interesting pathway for children to develop their calculation skills.

In conclusion, our results revealed that there is a relationship between sequence patterning in particular number, letter, time, and calculation. Furthermore, sequence patterning in the dimensions of number and time are strong predictors of children's calculation abilities above and beyond symbolic number comparison, motor processing speed, visuospatial working memory, and nonverbal IQ suggesting that certain types of sequence patterning make a significant contribution to individual differences in young children's arithmetic abilities.

\section{Acknowledgments}

We thank all the students, teachers, principals, and secretaries who participated in this research. Special thanks are due to Marinka Gadzichowski for her open communication and sharing of the patterning task used in this study. This work was partially supported by Grant C16/16/001 from University of Leuven Research Fund.

\section{Appendix}

\section{Calculation task}

\section{ADDITION}

\section{A}

$2+4=$

$1+2=$

$5+4=$

$3+1=$

$2+6=$

$7+3=$

B

$8+6=$

$5+11=$

$3+9=$

$13+6=$

$7+4=$

$12+3=$

C

$11+27=$

$76+23=$

$35+13=$

$41+26=$

$32+54=$

$24+31=$

\section{D}

$17+16=$

$35+26=$

$66+29=$

$49+22=$

$28+14=$

$27+58=$

E

$113+105=$

$453+536=$

$324+161=$

$404+273=$

$211+384=$

$534+262=$

F

$349+125=$

$108+167=$

$236+647=$

$513+178=$

$258+336=$

$568+424=$

\section{SUBTRACTION}

A

$3-2=$

$9-2=$

$6-4=$

$8-3=$

$5-1=$

$7-4=$

B

$15-2=$

$16-4=$

$17-6=$

$18-3=$

$19-8=$

$17-5=$

C

$97-16=$

$35-14=$

$66-12=$

$59-17=$

$89-23=$

$68-31=$

\section{D}

$56-38=$

$24-17=$

$92-13=$

$41-16=$

$87-18=$

$63-24=$

E

$778-355=$

$212-101=$

$979-323=$

$338-122=$

$686-124=$

$553-231=$

$\mathrm{F}$

$311-209=$

$883-325=$

$337-118=$

$923-288=$

$645-217=$

$462-126=$

\section{References}

Andraszewicz, S., Scheibehenne, B., Rieskamp, J., Grasman, R., Verhagen, J., \& Wagenmakers, E. J. (2015). An introduction to Bayesian hypothesis testing for management research. Journal of Management, 41, 521-543. 
Björklund, C., \& Pramling, N. (2014). Pattern discernment and pseudo-conceptual development in early childhood mathematics education. International Journal of Early Years Education, 22, 89-104.

Blanton, M. L., \& Kaput, J. J. (2005). Characterizing a classroom practice that promotes algebraic reasoning. Journal for Research in Mathematics Education, 36, 412-446.

Brankaer, C., Ghesquière, P., \& De Smedt, B. (2017). Symbolic magnitude processing in elementary school children: A group administered paper-and-pencil measure (SYMP Test). Behavior Research Methods, 49, 1361-1373.

Bueti, D., \& Walsh, V. (2009). The parietal cortex and the representation of time, space, number, and other magnitudes. Philosophical Transactions of the Royal Society B: Biological Sciences, 364, 1831-1840.

Carpenter, T. P., Levi, L., Franke, M. L., \& Zeringue, J. K. (2005). Algebra in elementary school: Developing relational thinking. Zentralblatt für Didaktik der Mathematik, 37, 53-59.

Chu, F. W., vanMarle, K., \& Geary, D. C. (2015). Early numerical foundations of young children's mathematical development. Journal of Experimental Child Psychology, 132, 205-212.

Clements, D. H., Sarama, J. H., \& Liu, X. H. (2008). Development of a measure of early mathematics achievement using the Rasch model: The research-based early maths assessment. Educational Psychology, 28, 457-482.

De Smedt, B. (2016). Individual differences in arithmetic fact retrieval. In D. Berch, D. Geary, \& K. Mann-Koepke (Eds.). Mathematical cognition and learning (Vol. 2, pp. 219-243). San Diego: Elsevier Academic Press.

De Smedt, B., Janssen, R., Bouwens, K., Verschaffel, L., Boets, B., \& Ghesquière, P. (2009). Working memory and individual differences in mathematics achievement: A longitudinal study from first grade to second grade. Journal of Experimental Child Psychology, 103, 186-201.

Desoete, A. (2015). Cognitive predictors of mathematical abilities and disabilities. In R. Cohen Kadosh \& A. Dowker (Eds.), The Oxford handbook of mathematical cognition (pp. 899-916). Oxford, UK: Oxford University Press.

Economopoulos, K. (1998). What comes next? The mathematics of pattern in kindergarten. Teaching Children Mathematics, 5 , 230-234.

Empson, S. B., Levi, L., \& Carpenter, T. P. (2011). The algebraic nature of fractions: Developing relational thinking in elementary school. In J. Cai \& E. Knuth (Eds.), Early algebraization (pp. 409-428). Heidelberg, Germany: Springer.

Ford, M. S., \& Crew, C. G. (1991). Table-top mathematics-A home-study program for early childhood. The Arithmetic Teacher, 38 (8), 6-12.

Fyfe, E. R., McNeil, N. M., \& Rittle-Johnson, B. (2015). Easy as ABCABC: Abstract language facilitates performance on a concrete patterning task. Child Development, 86, 927-935.

Gadzichowski, K. M. (2012). Examining patterning abilities in first grade children: A comparison of dimension, orientation, number of items skipped, and position of the missing item. Psychology, 3, 1177-1182.

Goffin, C., \& Ansari, D. (2016). Beyond magnitude: Judging ordinality of symbolic number is unrelated to magnitude comparison and independently relates to individual differences in arithmetic. Cognition, 150, 68-76.

Hendricks, C., Trueblood, L., \& Pasnak, R. (2006). Effects of teaching patterning to 1st-graders. Journal of Research in Childhood Education, 21, 79-89.

Kidd, J. K., Carlson, A. G., Gadzichowski, K. M., Boyer, C. E., Gallington, D. A., \& Pasnak, R. (2013). Effects of patterning instruction on the academic achievement of 1st-grade children. Journal of Research in Childhood Education, 27, 224-238.

Kidd, J. K., Pasnak, R., Gadzichowski, K. M., Gallington, D. A., McKnight, P., Boyer, C. E., \& Carlson, A. (2014). Instructing first-grade children on patterning improves reading and mathematics. Early Education E' Development, 25, 134-151.

Lee, K., Ng, S. F., Bull, R., Pe, M. L., \& Ho, R. H. M. (2011). Are patterns important? An investigation of the relationships between proficiencies in patterns, computation, executive functioning, and algebraic word problems. Journal of Educational Psychology, 103, 269-281.

Lee, K., Ng, S. F., Pe, M. L., Ang, S. Y., Hasshim, M. N. A. M., \& Bull, R. (2012). The cognitive underpinnings of emerging mathematical skills: Executive functioning, patterns, numeracy, and arithmetic. British Journal of Educational Psychology, 82, $82-99$.

Lourenco, S. F., \& Bonny, J. W. (2017). Representations of numerical and non-numerical magnitude both contribute to mathematical competence in children. Developmental Science. https://doi.org/10.1111/desc.12418 (Advance online publication).

Lyons, I. M., \& Beilock, S. L. (2011). Numerical ordering ability mediates the relation between number-sense and arithmetic competence. Cognition, 12, 256-261.

Lyons, I. M., \& Beilock, S. L. (2013). Ordinality and the nature of symbolic numbers. Journal of Neuroscience, 33, $17052-17061$.

Lyons, I. M., Vogel, S. E., \& Ansari, D. (2016). On the ordinality of numbers: A review of neural and behavioral studies. Progress in Brain Research, 227, 187-221.

Miller, M. R., Rittle-Johnson, B., Loehr, A. M., \& Fyfe, E. R. (2016). The influence of relational knowledge and executive function on preschoolers' repeating pattern knowledge. Journal of Cognition and Development, 17, 85-104.

Molina, M., Castro, E., \& Ambrose, R. (2005). Enriching arithmetic learning by promoting relational thinking. International Journal of Learning, 12, 265-270.

Napaphun, V. (2012). Relational thinking: Learning arithmetic in order to promote algebraic thinking. Journal of Science and Mathematics Education in Southeast Asia, 35, 84-101.

Papic, M. M., Mulligan, J. T., \& Mitchelmore, M. C. (2011). Assessing the development of preschoolers' mathematical patterning. Journal for Research in Mathematics Education, 42, 237-269.

Park, Y., \& Cho, S. (2017). Developmental changes in the relationships between magnitude acuities and mathematical achievement in elementary school children. Educational Psychology, 36, 873-887.

Pasnak, R., Kidd, J. K., Gadzichowski, K. M., Gallington, D. A., Schmerold, K. L., \& West, H. (2015). Abstracting sequences: Reasoning that is a key to academic achievement. Journal of Genetic Psychology, 176, 171-193.

Pasnak, R., Schmerold, K. L., Robinson, M. F., Gadzichowski, K. M., Bock, A. M., O’Brien, S. E., ... Gallington, D. A. (2016). Understanding number sequences leads to understanding mathematics concepts. Journal of Educational Research, 109, 640-646. 
Peng, P., Namkung, J., Barnes, M., \& Sun, C. Y. (2016). A meta-analysis of mathematics and working memory: Moderating effects of working memory domain, type of mathematics skill, and sample characteristics. Journal of Educational Psychology, 108, 455-473.

Piaget, J. (1952). The child's conception of number. New York: Humanities Press.

Rittle-Johnson, B., Fyfe, E. R., Loehr, A. M., \& Miller, M. R. (2015). Beyond numeracy in preschool: Adding patterns to the equation. Early Childhood Research Quarterly, 31, 101-112.

Rittle-Johnson, B., Fyfe, E. R., McLean, L. E., \& McEldoon, K. L. (2013). Emerging understanding of patterning in 4-year-olds. Journal of Cognition and Development, 14, 376-396.

Sarama, J., \& Clements, D. H. (2009). Building blocks and cognitive building blocks: Playing to know the world mathematically. American Journal of Play, 1, 313-337.

Sattler, J. M. (2001). Assessment of children: Cognitive applications (Vol. 4) San Diego: Author.

Schmerold, K., Bock, A., Peterson, M., Leaf, B., Vennergrund, K., \& Pasnak, R. (2017). The relations between patterning, executive function, and mathematics. Journal of Psychology: Interdisciplinary and Applied, 151, 207-228.

Schneider, M., Beeres, K., Coban, L., Merz, S., Schmidt, S., Stricker, J., \& De Smedt, B. (2017). Associations of non-symbolic and symbolic numerical magnitude processing with mathematical competence: A meta-analysis. Developmental Science. https:// doi.org/10.1111/desc.12372 (Advance online publication).

Starkey, P., Klein, A., \& Wakeley, A. (2004). Enhancing young children's mathematical knowledge through a pre-kindergarten mathematics intervention. Early Childhood Research Quarterly, 19, 99-120.

Thomas, N. D., Mulligan, J. T., \& Goldin, G. A. (2002). Children's representation and structural development of the counting sequence 1-100. Journal of Mathematical Behaviour, 22, 117-133.

Vanbinst, K., \& De Smedt, B. (2016). Individual differences in children's mathematics achievement: The roles of symbolic numerical magnitude processing and domain-general cognitive functions. Progress in Brain Research, 227, 105-130.

Warren, E. A., Cooper, T. J., \& Lamb, J. T. (2006). Investigating functional thinking in the elementary classroom: Foundations of early algebraic reasoning. Journal of Mathematical Behavior, 25, 208-223.

Wechsler, D. (1991). The Wechsler intelligence scale for children-third edition. San Antonio, TX: Psychological Corporation.

Xenidou-Dervou, I., De Smedt, B., van der Schoot, M., \& van Lieshout, E. (2013). Individual differences in kindergarten math achievement: The integrative role of approximation skills and working memory. Learning and Individual Differences, 28, 119-129. 